Article

\title{
Effects of Coupling Agents on the Structure and Electrical Properties of PZT-Poly (Vinylidene Fluoride) Composites
}

\author{
Rui Li, Liu Zhang, Zhiliang Shi and Jianzhong Pei * \\ Highway School, Chang'an University, Xi'an 710064, China; lirui@chd.edu.cn (R.L.); \\ liuzhang2015121190@chd.edu.cn (L.Z.); shizhiliang@chd.edu.cn (Z.S.) \\ * Correspondence: Peijianzhong@126.com; Tel.: +86-29-6263-0058 \\ Academic Editor: Andrew J. Ruys \\ Received: 14 June 2016; Accepted: 20 September 2016; Published: 3 October 2016
}

\begin{abstract}
Piezoelectric ceramic transducer (PZT)-Poly (vinylidene fluoride) composites were prepared by the hot-pressing method. Before addition, PZT particles were firstly modified with two different coupling agents. The micromorphology, microstructure, dielectric properties, and piezoelectric properties of the composites were characterized and investigated. Results indicated that PZT particles were homogeneously dispersed in the poly (vinylidene fluoride) (PVDF) matrix by the addition of coupling agents. The electric properties of PZT-PVDF composites with NDZ-101 were the best. Especially when the volume ratio of the titanate coupling agent NDZ-101 was 1\%, the piezoelectric strain constant $\mathrm{d}_{33}$ of PZT-PVDF composites reached maximum value $19.23 \mathrm{pC} / \mathrm{N}$; its relative dielectric constant $\varepsilon_{r}$ was 67.45; at the same time its dielectric loss tan $\delta$ was 0.0766 .
\end{abstract}

Keywords: PZT-PVDF; coupling agents; dielectric properties; piezoelectric properties

\section{Introduction}

Hybrid organic-inorganic materials have been produced by many methods, such as sol-gel. The influence of siloxane composition on properties of ployimide-silica hybrids has also been known. The piezoelectric transducer encouraged researchers to explore better piezoelectric materials. Then, the coupling agent went into public view. This paper carried out various research into two kinds of coupling agents.

As an engineering material, poly (vinylidene fluoride) (PVDF) had been extensively applied in many areas such as electric industries, microelectronics, acoustic impedance underwater, and so on [1]. During the past decade, increasing attention has been paid to the PVDF organic-inorganic composite materials, and it has been proved that the mechanical and electrical properties of PVDF hybrid films can be improved by incorporation of fillers such as carbon nanotube [2], silica [3-5], and titaniain to the pristine PVDF matrix [6,7].

Among these inorganic materials, piezoelectric ceramic transducer (PZT) power is often chosen as a filler to improve electric properties of the polymer materials due to its extremely high electric qualities [8-10]. These PZT/PVDF composites could be widely applied in microelectronics devices fields. However, due to the lack of mechanical resistance and no flexibility, the combination of PZT particles with PVDF in micro scale is very difficult [11-13]. One of the most important key points of PZT/PVDF hybrid composites is to improve the interface of PZT in the polymer matrix.

The coupling agents connected organic with inorganic materials and improve the compatibility between the two phases effectively $[14,15]$. However, little attention has been focused on the effects of different coupling agents on structure and properties of PZT/PVDF hybrid composites.

In this paper, two different coupling agents (silane coupling agent $\mathrm{KH}-570$ and titanate coupling agent NDZ-101) were used to improve the surface state of the PZT particles. The PZT/PVDF 
composites appear to be a good alternative for applications as sensors and actuators because they are capable of combining the better properties of ceramic and polymer. This work shows some results of preparation, characterizations and electric properties of composites, consisting of PVDF and PZT, with $0-3$ connectivity.

\section{Experimental Procedure}

\subsection{Materials}

PVDF powders $\left(\rho=1.77 \mathrm{~g} / \mathrm{cm}^{3}, T_{m}=167^{\circ} \mathrm{C}\right)$ with a mean particle size of about $3 \mu \mathrm{m}$ were provided by Shanghai 3F New Materials Ltd., Co., Shanghai, China. The applied pressure is $225 \mathrm{MPa}$. PZT ceramic $\left(\rho=7.6 \mathrm{~g} / \mathrm{cm}^{3}\right)$ were prepared via the conventional solid-state reaction method, which is supplied by Institute of Acoustics, Chinese Academy of Science, Shanghai, China. PZT powder with the average particle size of about 50 70 $\mu \mathrm{m}$ was obtained from crushed PZT disks. The 3-methacryl oxypropyl-trimethoxysilane (KH-570) coupling agents were purchased from $\mathrm{Xi}^{\prime}$ an Chemical Reagent Ltd., Co., Xi' an China. The isopropyl dioleic(dioctylphosphate) titanate (NDZ-101) coupling agents was purchased from Shandong Yuanhang Chemical Materials Ltd., Co., Jinan, China. All other chemicals and reagents were provided by Shanghai Experiment Reagent Ltd., Co., Shanghai, China.

\subsection{Preparation of PZT-PVDF Composites with Coupling Agents}

This test prepared different concentrations $(0 \%, 0.5 \%, 1.0 \%, 1.5 \%$ and $2 \%)$ of coupling agent solution with the treatment of ultrasonic wave. The purpose of preparing coupling agent liquid is to modify the PZT power. Firstly, PZT particles were absolutely dissolved in ethanol. Then, the mixture was heated up in a water bath of $80^{\circ} \mathrm{C}$. Then, it was stirred mechanically again for $3 \mathrm{~h}$ and heated up at $120^{\circ} \mathrm{C}$ for $12 \mathrm{~h}$. Finally, it was abraded to use. This experiment prepared modified PZT power by mixing coupling agent solution and PZT power. Calculated quantity of modified PZT particles with two coupling agent content $0 \%, 0.5 \%, 1.0 \%, 1.5 \%$ and $2 \%$ were mixed with PVDF. Next, this mixture was compressed into a disk of $13 \mathrm{~mm}$ in diameter and $1 \mathrm{~mm}$ in thickness. Next, we dried the disk in an oven and plated electrode on it. Then we polarized it for $30 \mathrm{~min}$ by placing it in constant temperature silicone oil under the condition of the polarization voltage for $3-5 \mathrm{kV} / \mathrm{mm}$, and temperature of $100{ }^{\circ} \mathrm{C}$. Finally, the samples were tested after static duration for $24 \mathrm{~h}$.

\subsection{Measurements}

All these measurements were collected under the condition of room temperature and atmospheric pressure. The internal microstructure of PZT-PVDF composites was observed by Scanning Electron Microscopy (SEM, JSM-6390A) (JEOL Ltd., Tokyo, Japan); the dielectric constant $\varepsilon_{r}$ and the dielectric loss $\tan \delta$ of PZT-PVDF composites were measured by a composites were measured by a Agilent 4294A Meter (Agilent Technologies Ltd., Tokyo, Japan) under $1 \mathrm{kHz}$; and the piezoelectric coefficient $\mathrm{d}_{33}$ was measured by a ZJ-3A piezo $\mathrm{d}_{33}$ tester (Institute of Acoustics, Chinese Academy of Science, Beijing, China).

\section{Results and Discussion}

\subsection{FTIR (Fourier Transform infrared spectroscopy)}

Figure 1 shows the Fourier Transform infrared spectroscopy (FTIR) spectra of PZT particles before and after treated respectively with coupling agent contents of $0 \%, 0.5 \%, 1 \%, 1.5 \%$, and $2 \%$. As shown in Figure 1, there is a stretching-vibration-absorption peak of functional groups of $\mathrm{CH}_{3}, \mathrm{CH}_{2}, \mathrm{CH}$ at $2929.7 \mathrm{~cm}^{-1}$. At the same time there exists a stretching vibration characteristic peak of $\mathrm{C}-\mathrm{O}-\mathrm{Si}$ at $1060.7 \mathrm{~cm}^{-1}$. All these peaks belonged to the characteristic peaks of KH-570. Further analysis of the results showed that the peak intensity at $761.8 \mathrm{~cm}^{-1}$ increased, and the peak became narrowing and sharp. The appearance of such absorption peaks showed that KH-570 had an effect on the surface of 
PZT particles. Figure $1 \mathrm{~b}$ shows the FTIR spectrum PZT ceramic particles treated and untreated by the titanate coupling agent NDZ-101, respectively. It can be found that the PZT treated by NDZ-101 showed an evident absorption peak of $\mathrm{CH}_{2}$ and $\mathrm{CH}_{3}$ at $2920.07 \mathrm{~cm}^{-1}$, which was the characteristic absorption peak of $\mathrm{CH}_{2}$ and $\mathrm{CH}_{3}$ functional group in NDZ-101. It showed NDZ-101 on the surface of PZT particles. In addition, it showed an absorption peak of Ti-O bond at $1076.22 \mathrm{~cm}^{-1}$, which had an overlap with the absorption peak of PZT, and the peak intensity was increased, which showed that the chemical reaction may occur between titanate coupling agent and PZT particles.
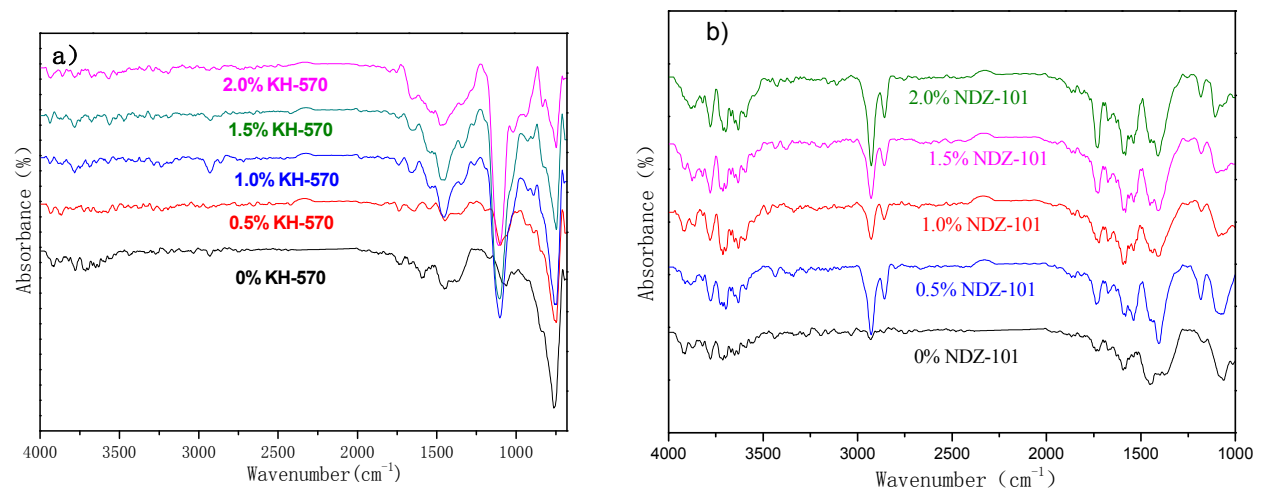

Figure 1. FTIR (Fourier Transform infrared spectroscopy) spectra of piezoelectric ceramic transducer (PZT) with surface treated and untreated by coupling agent.

\subsection{SEM (Scanning Electron Microscopy)}

Figure 2 shows the Scanning Electron Microscopy (SEM) images of PZT particles before and after $1 \%$ volume ratio coupling agent treatment in PZT-PVDF composites. Among them, Figure $2 \mathrm{~b}$ shows the SEM images of PZT particles treated by KH-570 in the PZT-PVDF composites. It can be seen that the PZT particle is equally distributed. Figure 2c shows the SEM images of PZT particles before and after 1\% (volume ratio) titanate coupling agent NDZ-101 treatment in PZT-PVDF composites. From the SEM image, it can be observed that the defects of composite were greatly reduced after treatment by NDZ-101, and the microstructure was compact and dense. Compared with Figure 2c, the spacing between PZT particles in Figure $2 b$ became larger, and their interface faintness increased. This was caused by the silane coupling agent acting as the role of "molecular bridge" between ceramic phase and polymer phase, which enhanced adhesion between the two phases so as to be linked closely and weaken the traces of the two phase interface.

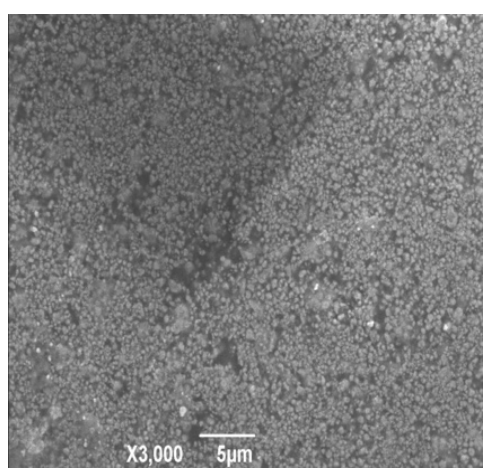

(a)

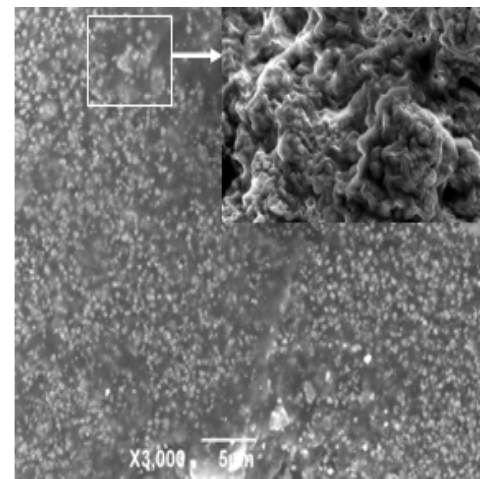

(b)

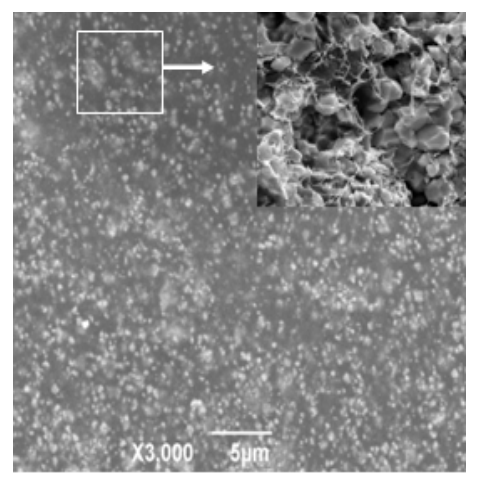

(c)

Figure 2. SEM (Scanning Electron Microscopy) of PZT-poly (vinylidene fluoride) (PVDF) composites. (a) Unmodified; (b) 1\% KH-570; (c) 1\% NDZ-101. 
The PZT particles were uniformly distributed in the PVDF without obvious defects. Moreover, PVDF infiltrates into the PZT ceramic material. Although the effect of KH-570 treatment is not obvious, the interface between PVDF and PZT is visible and clear. The results show clearly that there is no infiltration phenomenon between the PZT and PVDF. The interfacial properties in two phases were changed by NDZ-101, and the adhesion between the two phases in the PZT-PVDF composites was effectively improved.

\subsection{Effects of Coupling Agent on the Dielectric Properties of PZT-PVDF}

Figure 3 showed the dielectric properties versus volume percent of KH-570 in PZT/PVDF composites. The test frequency was at $1 \mathrm{kHz}$ under the condition of room temperature. As can be seen, as the content of KH-570 increased, the relative dielectric constant $\varepsilon_{r}$ of PZT-PVDF composites decreased gradually. Without silane coupling agent KH-570, the relative dielectric constant $\varepsilon_{r}$ of PZT-PVDF composites was 54.75. When the content of the KH-570 was $2.0 \%$, the relative dielectric constant $\varepsilon_{r}$ was reduced to 36.38 , which was reduced by $33.55 \%$. Meanwhile, the dielectric loss tan $\delta$ of the composites increased with the increased content of KH-570. By analysis, after PZT-PVDF composites were treated by $\mathrm{KH}-570$ coupling agent, the infiltration effect of the ceramic phase in the polymer phase was improved, and the structures of the PZT-PVDF composites became more compact, and the porosity was decreased, all of which enhanced the dielectric constant of the PZT-PVDF composites to some extent. What cannot be overlooked was that a layer of insulation was formed on the surface of the ceramic phase which was caused by PVDF molecular chain and $\mathrm{KH}-570$ wrapped. The greater the $\mathrm{KH}-570$ content was, the greater the insulating layer thickness was. However, the dielectric constant of the insulating layer was smaller than the ceramic phase and polymerization phase. Therefore, the greater the content of KH-570 there was, the smaller the values of the dielectric constant the PZT-PVDF composites had. Simultaneously, multi molecular layers which were caused by KH-570 to improve the ceramic phase interface, declined the uniformity degree of the PZT-PVDF composites interface and increased the relaxation polarization current. Finally, all these lead to an increase of the dielectric loss tan $\delta$ of PZT-PVDF composites.

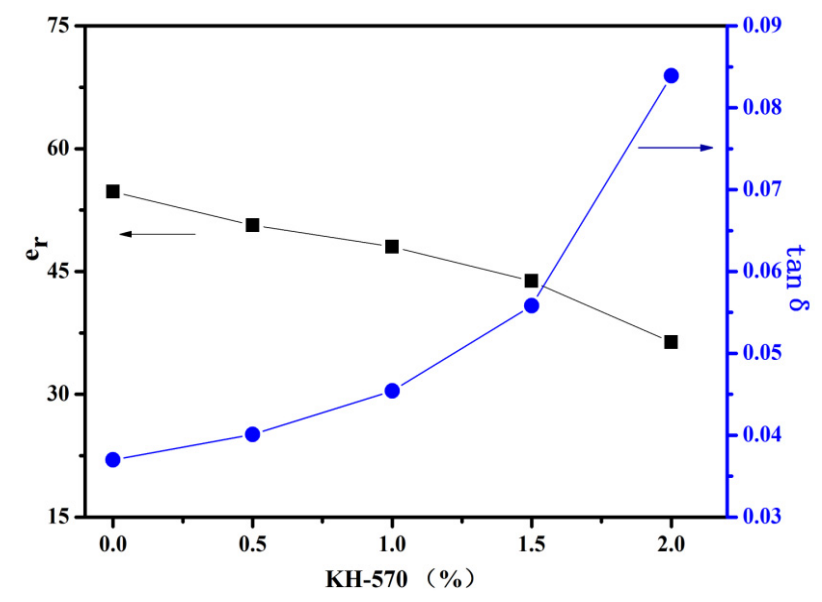

Figure 3. Effects of $\mathrm{KH}-570$ on the dielectric properties of the PZT-PVDF.

Figure 4 showed the dielectric constant versus volume percent of NDZ-101 in 0.5PZT/0.5PVDF composites. The test frequency was at $1 \mathrm{kHz}$ under the condition of room temperature. As can be seen from Figure 4, as the NDZ-101 content was increased, the relative dielectric constant $\varepsilon_{r}$ of the PZT-PVDF increased slightly. In particular, when the content was $0.5 \%$, the dielectric constant $\varepsilon_{r}$ was increased from the initial 64.75 to a maximum of 69.29 , i.e., it increased only by $7.01 \%$. However, as the NDZ-101 continued to be increased, the relative dielectric constant $\varepsilon_{r}$ of the PZT-PVDF composites decreased. Simultaneously, with the increase of content, the dielectric loss $\tan \delta$ of the PZT-PVDF 
composites showed an increasing trend. When the content was 0 , the dielectric loss $\tan \delta$ was 0.037 . When the content was $2 \%$, the dielectric loss reached 0.1175 . By analysis, during the preparation of piezoelectric composites, adding a certain amount of NDZ-101 improved the two-phase interface state, but the effect was not obvious. Meanwhile, NDZ-101 can lower the dielectric properties of PZT-PVDF. In contrast, the dielectric loss tan $\delta$ of the PZT-PVDF composites increased rapidly. This was because NDZ-101 produced an accumulation phenomenon and reduced the interfacial strength greatly.

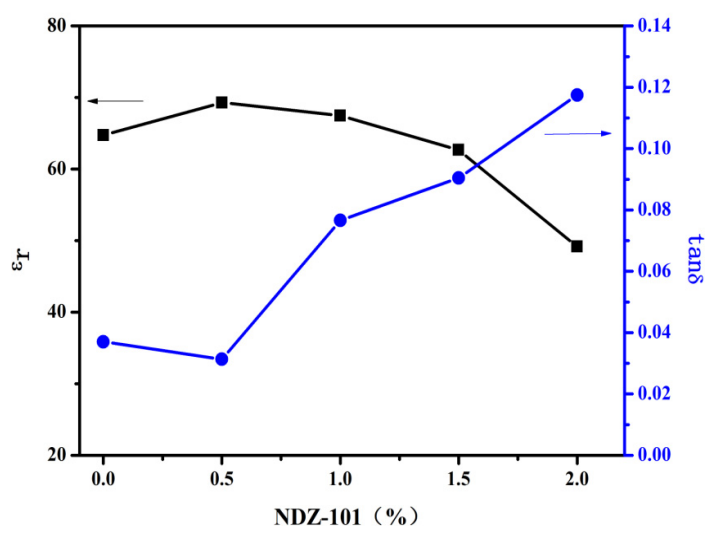

Figure 4. Effects of NDZ-101 on the dielectric properties of PZT-PVDF.

\subsection{Effects of Coupling Agent on the Piezoelectric Properties of PZT-PVDF}

Figure 5 showed the piezoelectric constant $d_{33}$ and $g_{33}$ versus volume percent of $\mathrm{KH}-570$ in the PZT-PVDF composites. It indicated that the more contents of $\mathrm{KH}-570$ there was, the smaller the values of the piezoelectric strain constant $\mathrm{d}_{33}$ and the piezoelectric voltage constant $\mathrm{g}_{33}$ of the PZT-PVDF composites were. According to the abovementioned SEM, although KH-570 enhanced the adhesion of the two phases in the PZT-PVDF composites, it also added a layer of insulator on the surface of the ceramic, which separated the conductive path internal composites and reduced the conductivity. All these ultimately reduced the performance of the piezoelectric properties of the PZT-PVDF.

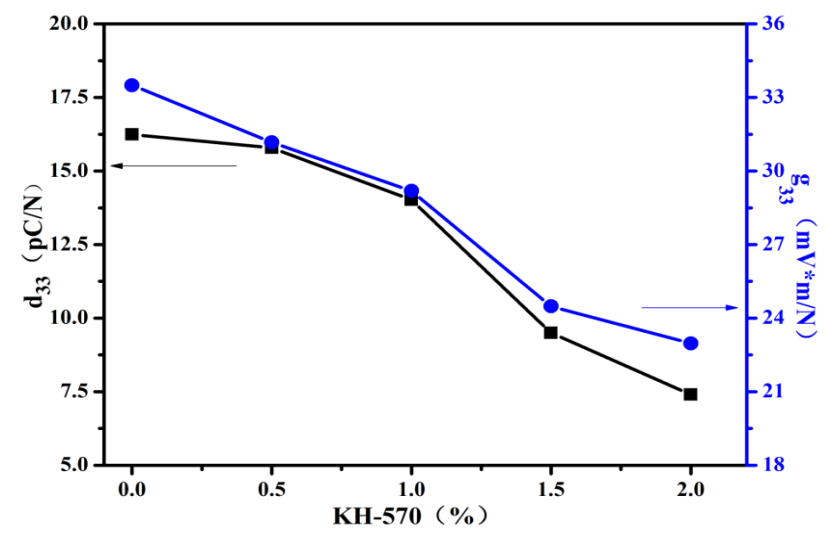

Figure 5. Effects of KH-570 on the piezoelectric properties of PZT-PVDF.

Figure 6 showed the piezoelectric constant versus volume percent of NDZ-101 in PZT-PVDF composites. As shown in Figure 6, as the content of NDZ-101 increased, the piezoelectric strain constant $\mathrm{d}_{33}$ of the PZT-PVDF composites firstly increased gradually and then decreased rapidly. The best content of NDZ-101 was $1 \%$. Meanwhile, the $\mathrm{d}_{33}$ of the PZT-PVDF composites reached the maximum $19.23 \mathrm{pC} / \mathrm{N}$. It was easy to find that with NDZ-101 coupling agent, the trends of piezoelectric voltage constant $g_{33}$ were similar to the $\mathrm{d}_{33}$. The $\mathrm{g}_{33}$ of the PZT-PVDF composites reached the maximum $33.54 \mathrm{mV} \cdot \mathrm{m} / \mathrm{N}$ when the NDZ-101 was $1 \%$. 


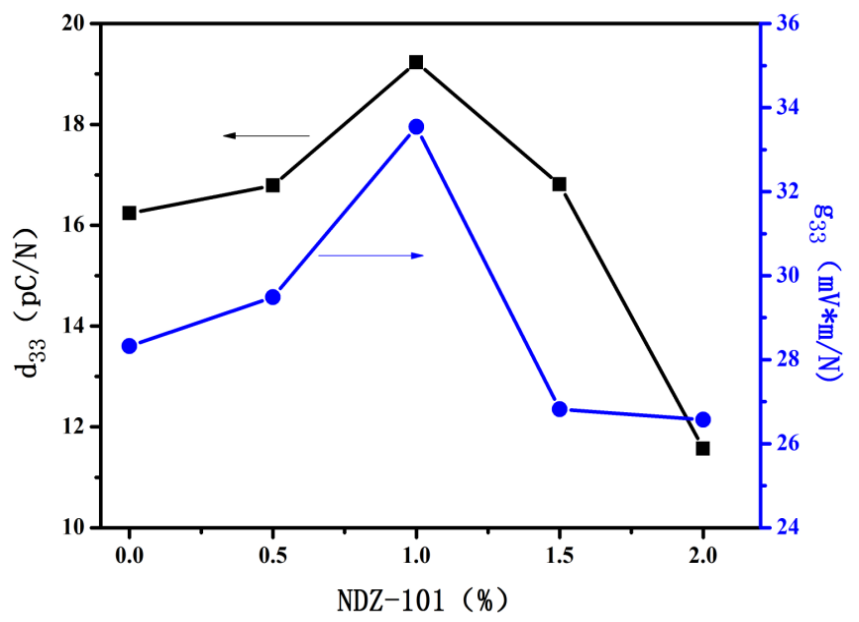

Figure 6. Effects of NDZ-101 on the piezoelectric properties of the PZT-PVDF.

\section{Conclusions}

The type and content of coupling agents had a great impact on the micro-structure and electrical properties of the PZT-PVDF composites. By the microscopic analysis, to some extent, these two kinds of coupling agents both optimized the two-phase interface state of the PZT-PVDF composites. However, with the addition of KH-570, the dielectric and the piezoelectric properties of PZT-PVDF composites both decreased, while the dielectric loss increased. The coupling agent NDZ-101 can improve the electrical properties of the PZT-PVDF composites, and the optimal content was $1 \%$. Under this condition, the relative dielectric constant $\varepsilon_{r}$ was 67.45 , the piezoelectric strain constant $\mathrm{d}_{33}$ was $19.23 \mathrm{pC} / \mathrm{N}$, the piezoelectric voltage constant $\mathrm{g}_{33}$ was $33.54 \mathrm{mV} \cdot \mathrm{m} / \mathrm{N}$, and the dielectric loss was 0.0766 .

Acknowledgments: The research was supported by the National Natural Science Foundation of China (Grant No. 51378073 and 51408048), the Key Program for International Science and Technology Cooperation Projects of Shaanxi province (Grant No. 2014KW10-03 and 2012KW-09), the China Postdoctoral Science Foundation (Grant No. 2015M570805 and 2016T90880), the Fundamental Research Funds for the Central Universities of Chang'an University (Grant No. 310821153502 and 310831151085$)$. Thank Hujun Liu for performing experiments.

Author Contributions: Rui Li and Jianzhong Pei conceived and designed the experiments; Zhiliang Shi and Liu Zhang analyzed the data; Rui Li and Liu Zhang wrote the paper.

Conflicts of Interest: The authors declare no conflict of interest.

\section{References}

1. Mascia, L.; Kioul, A. Influence of siloxane composition and morphology on properties of polyimide-silica hybrids. Polymer 1995, 36, 3649-3659. [CrossRef]

2. Rubia, L.; Vasconcelos, F.S.; Wander, L. Synthesis of titania-silica materials by sol-gel. Mater. Res. 2002, 5, 497-502.

3. Liu, L.Z.; Weng, L.; Song, Y.X.; Gao, L.; Lei, Q.Q. The effects of coupling agents on the properties of Polyimide/Nano- $\mathrm{Al}_{2} \mathrm{O}_{3}$ Three Layer Hybrid Films. J. Nanomater. 2010, 2010. [CrossRef]

4. Kim, D.W.; Lee, D.H.; Kim, B.K. Nanocomposite films. Macromol. Rapid Commun. 2006, 27, 1821-1825. [CrossRef]

5. Bharti, V.; Kaura, T.; Nath, R. Ferroelectric hysteresis in simultaneously stretched and corona-poled PVDF film. IEEE Trans. Dielectr. Electr. Insul. 1997, 4, 738-741. [CrossRef]

6. Savakus, H.P.; Klicker, K.A.; Newnham, R.E. PZT-Epoxy piezoelectric transducers: A simplified fabrication procedure. Mater. Res. Bull. 1981, 16, 677-680. [CrossRef]

7. Bayer, I.S.; Biswas, A.; Megaridis, C.M. Biocompatible poly vinylidene fluoride cyanoacrylate composite coatings with tunable hydrophobicity and bonding strength. Appl. Phys. Lett. 2008, 93. [CrossRef] 
8. Li, R.; Pei, J.Z.; Sun, C.L. Effect of nano-ZnO with modified mofified surface on properties of bitumen. Constr. Build. Mater. 2015, 98, 656-661. [CrossRef]

9. Wise, S.A. Displacement Properties of RAINBOW and THUNDER Piezoelectric Actuators. Sens. Actuators A Phys. 1998, 69, 33-38. [CrossRef]

10. Gao, Q.; Scheinbeim, J.I. Dipolar intermolecular interactions, structural development, and electromechanical properties in ferroelectric polymer blends of nylon and poly (vinylidene fluoride). Macromolecules 2000, 33, 7564-7572. [CrossRef]

11. Amash, A.; Zugenmaier, P. Thermal and dynamic mechanical investigations on fiber reinforced polypropylene composites. J. Appl. Polym. Sci. 1997, 63, 1143-1154. [CrossRef]

12. George, S.; Varughese, K.T. Dynamic mechanical properties of isotactic polypropylene/nitrile rubber blends: Effects of blends ratio, reactive compatibilization and dynamic vulcanization. J. Polym. Sci. Part B Polym. Phys. 1997, 35, 2309-2327. [CrossRef]

13. Kuang, D.L.; Li, R.; Pei, J.Z. Polyamide 11/Poly(vinylidene fluoride)/VinylAcetate-Maleic Anhydride Copolymer as Flexible Materials for Capacitors. Polymers 2014, 6, 2146-2156. [CrossRef]

14. Nwabunma, D.; Kyu, T. Polyolefin Blends; John Willey \& Sons, Inc.: Hoboken, NJ, USA, 2008.

15. Li, R.; Pei, J. High Dielectric Performance of Polyamide11/Poly (vinylidene fluoride) Blend Films Induced by Interfacial Glycidyl Methacrylate. Polym. Sci. Ser. A 2015, 57, 792-798. [CrossRef]

(c) 2016 by the authors; licensee MDPI, Basel, Switzerland. This article is an open access article distributed under the terms and conditions of the Creative Commons Attribution (CC-BY) license (http:/ / creativecommons.org/licenses/by/4.0/). 